\title{
Interactive comment on "Metamorphism of Arctic marine snow during the melt season. Impact on albedo" by Gauthier Verin et al.
}

\section{Gauthier Verin et al.}

gauthier.verin@takuvik.ulaval.ca

Received and published: 17 October 2019

Comment from author's Referee

My overall impression for the manuscript is that the first half part (âLijSection 3.3) is detailed but the latter half part is redundant. Although Chapter 3 is "Results", causes of temporal variations of albedos are DISCUSSED with sensitivity tests using radiative transfer model in Section 3.4. In "4 Discussion", similar things as described in "3 Results" are described repeatedly.

Another reason I feel redundant is that each of Sections 3.4, 4.1, 4.2 and 4.3 and Chapter 5 consists of only one paragraph although those paragraphs have a volume more or less one page. These make the findings, discussing points and conclusion 
of this paper ambiguous. It is needed to explain with a focus on important points and organize the text.

Author's response

The authors agree with the referee and find his comment pertinent. We voluntary decided to bring some discussion elements in section 3.4 (results) in order to introduce and justify, first the sensitivity study of SSA on albedo simulations (Figure 8), second the importance of the snow properties at the very surface (Figure 9) and third the effects of a varying ice albedo beneath the snowpack (Figure 10). We agree that these choices makes the discussion section redundant.

Changes in manuscript

The result section 3.4 will be reorganized. Only key elements needed to introduce the further snow albedo simulations (listed above) will remain in this section. The others elements will be moved to section 4.2. In addition, the discussion section will be divided into independent paragraphs in order to improve the readability and to point out the main focus and conclusions.

\section{Comment from author's Referee}

In Section 3.4, it is discussed on uncertainties in measurements of vertical snow physical properties as cause of disagreement in albedo between measurement and calculation in phases I and II. However, it is not discussed on the horizontal heterogeneity. This would be possible cause of the disagreement. Please discuss on this issue.

\section{Author's response}

We think that some main discrepancies between albedo measurements and simulations are indeed caused by high vertical heterogeneity of snow properties during phase I and II. During these periods spatial variability was very low. Especially during the surface melting period where surface changes (melt, fresh snow) were homogeneous over the snow cover. Remarkably, spatial variability was slightly higher during the first 
phase. Generally, the thickest dunes showed lower albedo than places around because they were not covered by fresher windslabs due to their height. These considerations did not stand anymore for phase III where spatial variabilities played a larger role.

Changes in manuscript

The above details have to be added into the text.

Comment from author's Referee

p.5, L6-8 "The correction concerning the determination of SSA of wet snow introduced by Gallet et al. (2014b) was not applied in this study because it did not induce significant changes on albedo simulations at the end.": Please indicate amount of error in SSA by not applying this correction.

\section{Author's response}

The corrected SSA were calculated using high liquid water contents, higher than reasonably observed on the field. The corrections were not significant and did not explain the deviations between simulated and observed albedo.

Changes in manuscript

The amount of error in SSA by not applying this correction and the corresponding water contents used will be added to the text.

\section{Comment from author's Referee}

p.6 L4 "Albedo spectra were finally smoothed using a low-pass filter.": Does this mean the raw spectral albedo varies not smoothly unless a low-pass filter? Please explain the reason.

Author's response

The raw albedo spectra were indeed noisy. The noise was apparently related to the temperature of the spectrometer we used. Data from 2016 campaign were more af- 
fected because of the warmer conditions. In addition, we did not use the same spectrometer in both field campaign (but same brand and version).

Changes in manuscript

These details will be added to the text in section 2.5.

Comment from author's Referee

p.10, L22-25 "If it is the case, . . .": I don't understand this discussion on snow impurities. It is described "the impurities may have potentially lowered the albedo by $1 \%$ at maximum . .." which is larger than the values of "reducing the reflected irradiance by $0.4 \%$ to $0.7 \%$ ". If effects caused by snow thickness variations is larger than the impurities effect (1\%), the latter (impurity effect) is not negligible.

Author's response

In the related section we aim to investigate the constant bias $(\sim 1.3 \%)$ between the observed and simulated albedo, especially during phase I (where the measurements are the most trustful). We concluded that this bias is likely due to both, the shadows generated by the operator and the equipment and the presence of snow impurities. Since we focus our study on the effects of SSA and snow thickness, the latter largely affecting the albedo at shorter wavelength during phase III (Figure 10). It appears that the impurity content are negligible compared to snow thickness in this wavelength range and then are not incorporated into the modeling.

Changes in manuscript The text ill be arranged and clarified to avoid any misunderstanding from the reader.

Comment from author's Referee

Printer-friendly version

4.1 Snowpack formation: This section describes observation results of snow physical properties qualitatively, some of which were shown in Sections 3.1 and 3.2. Dune formations continues from explanations for phases I - III in the first part, that is confusing. 
At least discussion on dunes should be made in separate paragraph.

Author's response

The authors are very attached to the reconstruction of the snowpack history based on snow physical properties sampled months after snow layers formations. However, we agree that the corresponding paragraph is confusing and has to be reformulated.

Interactive

comment

Changes in manuscript

The section will be reorganized and divided into relevant paragraphs in order to improve the readability. In addition, redundant elements presents in both sections (result and discussion) will be reduced to a minimum.

Interactive comment on The Cryosphere Discuss., https://doi.org/10.5194/tc-2019-113, 2019. 